\title{
The need to establish a cleft palate teaching program for residents and practicing orthodontists (An on-line cleft palate teaching program): www.cleftlippalateaudiovisuallecture.org
}

\author{
Samuel Berkowitz, DDS, MS, FICD ${ }^{1,2 *}$ \\ ${ }^{1}$ Adjunct Professor, Cleft Palate/Craniofacial Program, Department of Orthodontics, University of Illinois College of Dentistry, Chicago, USA \\ ${ }^{2}$ Department of Pediatrics and Surgery (Retired), University of Miami School of Medicine, Miami, USA
}

In order to achieve more successfully treated cleft lip and palate cases and obtain all treatment goals of good dental occlusion, facial aesthetics, speech and psychosocial development, it is necessary that more orthodontists become involved with the study and guidance of the growth and development from birth to adolescence. As a result of special training their role includes diagnosis of changing facial morphology and jaw function as a result of the anomaly, followed by its influence in growth. They provide orthodontic/orthopedic treatment and general expertise for consultation with all of the other members of the cleft and craniofacial team. Orthodontists are involved in one way or another with virtually all of the treatment procedures provided by all of the cleft palate-craniofacial team's specialists. Since palatal clefts vary in the extent of osteogenic and muscular deficiencies, surgeons should recognize that all clefts, although similarly classified, are not the same. Therefore, each case requires differential diagnosis and treatment planning. What may be the treatment of choice for one patient may be different for another even with the same cleft type.

The general aim of the cleft palate lecture is to present recognized treatment concepts from the clinical sciences of dentistry, medicine, speech, audiology, psychology, genetics, biology and ethics so that all aspects of treatment of cleft palate and other craniofacial anomalies can be scrutinized from long term clinical experience using objective treatment records of serial casts, lateral cephs and photographs.

The lecture examines the face with a cleft in all aspects as a biologic continuum from birth through post-natal growth and development to maturity at various stages of treatment.

In the past several decades many advances have taken place in cleft habilitation procedures. Unfortunately, many of these changes have not fulfilled all of their stated objectives and in some instances, these procedures were found to be either injurious or at best unnecessary. These errors need to be discussed in detail.

There are numerous types of congenital craniofacial anomalies, the most common of which is cleft lip and palate. In the United States, this birth defect affects approximately one in 650 newborns each year. Approximately one-half of these infants have associated malformations, either minor or major, according to conjunction with the cleft. Although the incidence figures for more complete anomalies or syndromes such as Apert syndrome, Crouzon disease, mandibular dysostosis or hemifacial microsomia are much lower than that for cleft lip and palate, the impact of craniofacial birth defects must be viewed in terms of the aggregate effect rather than the impact of any single entity. The impact is twofold: that on the patient and family and that on society.

Topics Covered:

- Embryopathogenesis of cleft palate development

- The natural history of cleft palate growth and development from birth to adolescence (with or without presurgical orthopedics)

- Complete bilateral cleft lip and palate

o Complete unilateral cleft lip and palate

o Isolated cleft palate

- Use and abuse of pre-surgical orthopedics with/without gingivoperisteoplasty

- The velopharyngeal mechanism and variations in the pharyngeal architecture which influences nasal air flow

- The use of a pharyngeal flap $v s$. pharyngioplasty to control air flow

- Timing of palatal closure as it influences occlusion, speech, and facial growth

- The use of a protraction facial mask to avoid mid-facial surgical advancement

- Craniofacial surgery and distraction osteogenesis

- The course also includes an online version of the book Cleft Palate Treatment: The Road to Normalcy (200 pages). Contents include:

o When a Child Is Born with a Cleft

Correspondence to: Samuel Berkowitz, DDS, MS, FICD, 11035 Paradela Street, COral Gables, FL.33156, USA, E-mail: sberk3140@aol.com

Received: June 28, 2015; Accepted: August 03, 2015; Published: August 09, 2015 
o

o The Anatomy of Clefts

o The Causes of Clefting

o The Cleft Palate Team

o Surgery

o $\quad$ Facial and Dental Concerns

o Preparing for Surgery

o Speech Concerns

This on-line lecture which can be seen at www.cleftlipplate.org or www.cleftlippalateaudiovisuallecture.org brings together treatment concepts from clinicians from the United States, Asia and Europe each of whom, in their own way, have been seeking answers to the multifaceted problem of cleft palate, regarding the embryopathogenesis, craniofacial growth, maxillary orthopedics, surgery, protraction of the maxilla, dental prostheses, secondary alveolar bone grafting, speech, hearing, genetics, psychosocial development and craniofacial surgery. The conclusions reached are the result of documented cases used in well-controlled clinic research that has withstood the test of review and re-examination.

It is my hope that more students and practicing orthodontists through a better understanding of the cleft palate defect and face will become involved in this field to be better able to evaluate present-day treatment practices and concepts to better plan their own treatment procedures.

Copyright: $@ 2015$ Berkowitz S. This is an open-access article distributed under the terms of the Creative Commons Attribution License, which permits unrestricted use, distribution, and reproduction in any medium, provided the original author and source are credited. 